\title{
ACTIVITY IN THE NUCLEI OF SEYFERT GALAXIES \\ IN THE VISUAL AND INFRARED
}

\author{
A. G. PACHOLCZYK
}

Steward Observatory, Tucson, Ariz., U.S.A.

\begin{abstract}
This paper reports (1) a possible $5.1 \mathrm{yr}$ period in the optical variations of the nucleus of NGC 4151 ; and (2) variations with a time-scale of a few days in the 2.2 micron flux density of NGC 1068, implying that the size of the infrared emitting region is not larger than a few times $10^{15} \mathrm{~cm}$.

I would like to communicate two results of photometric investigations, concerning the activity in the nuclei of the Seyfert galaxies.

First is the possibility of presence of a $5.1 \mathrm{yr}$ period in the optical variations of the nucleus of NGC 4151. This possibility is not incompatible with the analysis of data
\end{abstract}
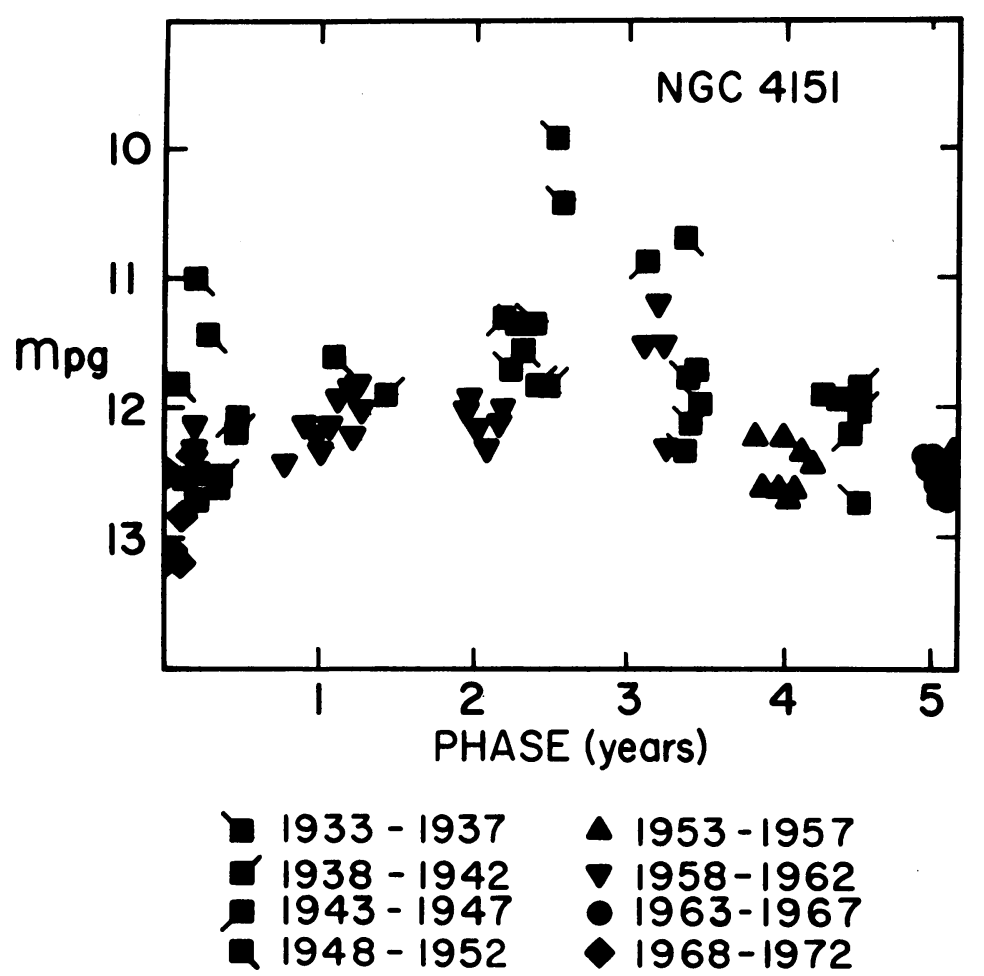

Fig. 1. The photographic magnitude of NGC 4151 as a function of phase within a $5.1 \mathrm{yr}$ period.

from Harvard patrol plates taken during the years 1932-1952 and from Steward plates taken between the years 1956 and 1968. Detailed description of the photometric data, procedure, errors and results is given in the paper by Pacholczyk (1971). Figure 1 represents the photographic magnitude of NGC 4151 as a function of phase 
within a $5.1 \mathrm{yr}$ period. Note a fairly well defined lower envelope of points. The amplitude of this long term change is about $1.5 \mathrm{mag}$. On this long term variation are superimposed short term changes with a similar amplitude but a time-scale of months (observed photoelectrically since 1967). The available data, summarized in Figure 1, are, of course, insufficient to make a definite statement about periodicity, and any more sophisticated statistical analysis of those data will not be meaningful at the present stage except, perhaps, for pointing out that the average magnitude during half-cycles between the minima is $11.64 \pm 0.12$ while the magnitude during the halfcycles around the minima is $12.34 \pm 0.05$. The Harvard plates used for measurements reported in Figure 1 were exposed $1-\frac{1}{2} \mathrm{~h}$ or more and contained a star-like image of NGC 4151 well above the plate limit. Efforts are being currently made to extend the measurements to shorter exposure plates, and to plates of other than RH series, which are abundant in the Harvard collection, and which will permit an analysis of the variability as far back as 1898 .

The $5.1 \mathrm{yr}$ period might be a manifestation of the presence of a rotating single massive object in the central region of the nucleus of NGC 4151. The possibility of the existence of 'spinars' in quasi-stellar objects and galactic nuclei was pointed out by Morrison (1969), Woltjer (1970), Cavaliere et al. (1969) while 'magnetoids' were invoked by Ozernoy (1966) and Piddington (1970). The object in NGC 4151, with a mass of the order of $2 \times 10^{9} M_{\odot}$, rotating with the angular velocity $\Omega$ of $4 \times 10^{-8} \mathrm{~s}$, would have a radius larger than the Schwarzschild radius of $5 \times 10^{13} \mathrm{~cm}$ but smaller than $\Omega / c=8 \times 10^{17} \mathrm{~cm}$. The emission of variable optical radiation is clearly not in the form of pulses, as in the quasistellar source 3C 345, but exhibits secondary changes with a time-scale of months due to some mechanism not necessarily connected with the rotation.

The second result is the presence of infrared variations of flux at 2.2 microns ( $\mathrm{K}$ photometric system) of the Seyfert galaxy NGC 1068 with a time-scale of a few days, implying that the size of the infrared emitting region is not larger than a few times $10^{15} \mathrm{~cm}$. Figure 2 summarizes the observations; Figure 3 describes the magnitude of photometric errors involved by displaying standard deviations of an observation of a comparison star and of NGC 1068. The deviations for NGC 1068 are significantly higher than if they were solely due to photometric errors. A detailed description of the photometric data and procedures, as well as a discussion of the accuracy of

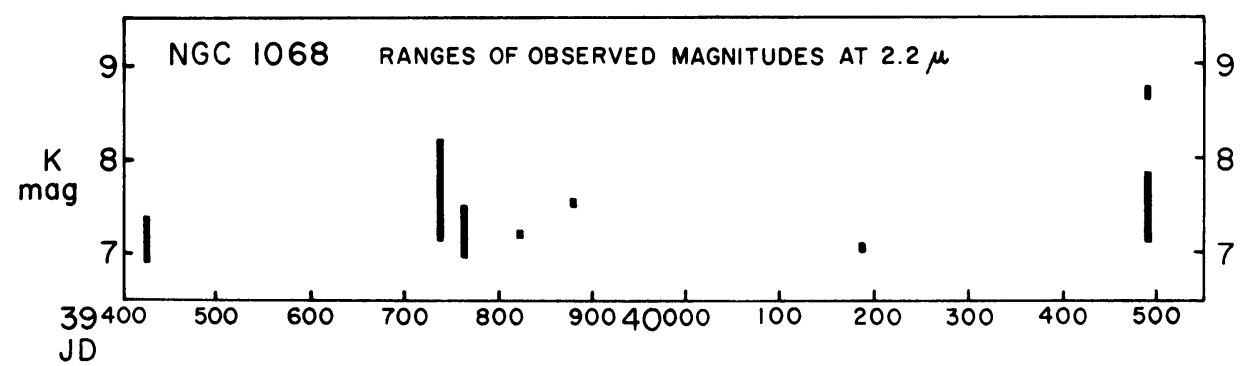

Fig. 2. Ranges of magnitudes of NGC 1068 at $2.2 \mu$. Time is expressed in Julian days. 
measurements and of differential extinction and other effects possibly contributing to the large scatter of data for NGC 1068, is contained in the paper by Pacholczyk (1970). A discussion of the role of the diaphragm centering errors, based on recent data on the distribution of brightness at $2.2 \mu$ in NGC 1068 obtained by G. Neugebauer, is given in the Appendix. Observations of NGC 4151 do not rule out possible variability of the infrared flux at $2.2 \mu$ (Figure 3); it is however clear that observations with more sensitive instrumentation are needed definitely to establish the variability of NGC 4151.

The limit on the size of the emitting region in NGC 1068, imposed by infrared variability, is incompatible with the interpretation that the observed infrared radiation is emitted by dust grains which absorb energy from a central compact optical or ultraviolet source. The $2.2 \mu$ emitting region must be at a distance of the order of $10^{18} \mathrm{~cm}$ from the central source and have a size of the same order of magnitude for any type of dust grains with an absorption efficiency not far below unity at ultraviolet and optical frequencies. A homogeneous synchrotron model, or even more, a model consisting of a large number of synchrotron sources ('irtrons'), cannot reconcile an emitting region as small as a few times $10^{15} \mathrm{~cm}$ with the lack of a self-absorption feature observed up to $100 \mu$ without incurring inverse Compton losses substantially exceeding the electron energy losses due to synchrotron radiation. The infrared characteristics of NGC 1068 could be explained in terms of a non-uniform synchrotron model with the field decreasing outwards, somewhat similar to that discussed by Rees and Sciama (1966) for 3C 273. The infrared radiation of a given wavelength would originate predominantly in a region at a certain distance from the center of a spherically-symmetric model; at smaller distances the source would be optically thick

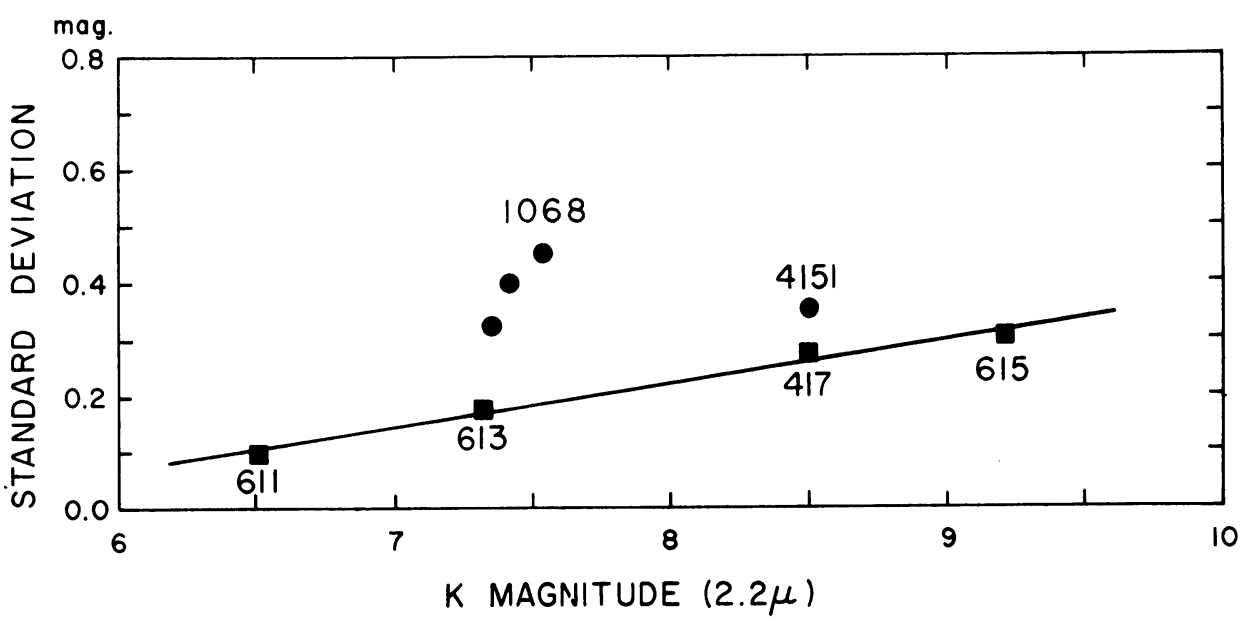

Fig. 3. Standard deviation from mean value of a single observation consisting of 20 differences between measurements of 'star' and of 'sky', each measurement employing an integration time of $15 \mathrm{sec}$. Squares represent observations of comparison stars of various magnitudes at $2.2 \mu$. Dots refer to measurements of the Seyfert galaxies NGC 1068 (the lower dot refers to all 33 observations of NGC 1068, the upper one represents observations on JD 39737, and the middle observations on JD 40487-90) and NGC 4151. 
to radiation of that and longer wavelength; radiation of longer wavelength would be emitted mainly at larger distances. The details of the model are being computed by W. G. Fogarty in his dissertation.

\section{Acknowledgement}

The work reported here was supported by the National Science Foundation under grant GP-9616.

\section{References}

Cavaliere, A., Pacini, F., and Setti, G.: 1969, Astrophys. Letters 4, 103.

Morrison, P.: 1969, Astrophys. J. Letters 157, L73.

Ozernoy, L. M.: 1966, Astron. Zh. 43, 300.

Pacholczyk, A. G.: 1970, Astrophys. J. Letters 161, L207.

Pacholczyk, A. G.: 1971, Astrophys. J. 163, 449.

Piddington, J. H.: 1970, Monthly Notices Roy. Astron. Soc. 148, 131.

Rees, M. J. and Sciama, D. W.: 1966, Nature 211, 805.

Woltjer, L.: 1970, Columbia Astrophys. Lab. Contr. No. 24.

\section{Appendix}

\section{Diaphragm Centering Errors in K Photometry of NGC 1068}

Dr G. Neugebauer kindly communicated the results of his observations of NGC 1068 at $2.2 \mu$ with diaphragms of sizes ranging from $2^{\prime \prime}$ to $20^{\prime \prime}$ obtained in December 1969.

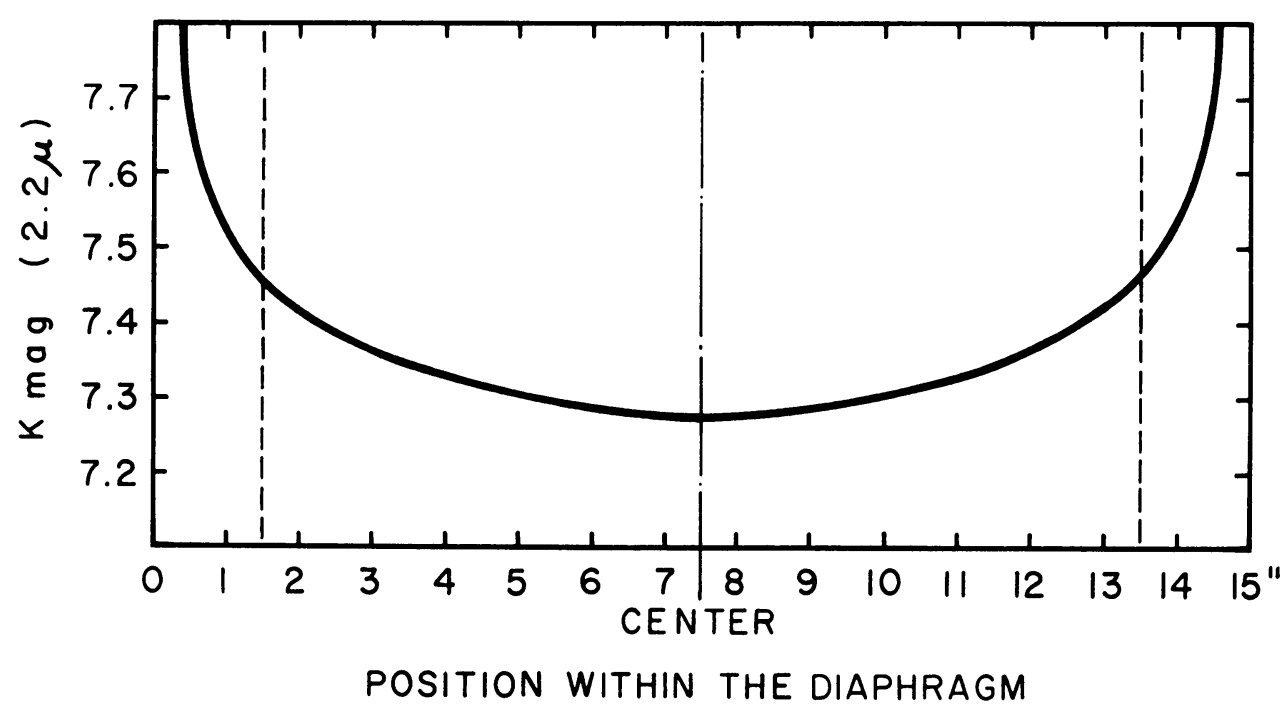

Fig. 4. The K magnitude of NGC 1068 as a function of the position of the center of the galaxy along the diameter of a circular diaphragm of $15^{\prime \prime}$. The difference in the $\mathrm{K}$ magnitudes for any position between the dotted lines and the central position is smaller than the total photometric error of measurement of the $\mathrm{K}$ magnitude of a point-like object (star) of comparable magnitude. 
His data permitted us to calculate the photometric errors due to improper centering of this object within the photometer diaphragm. In the paper 'Infrared Variability of the Seyfert Galaxy NGC 1068' (Pacholczyk, 1970) variations in the $2.2 \mu$ flux from this object are reported; the measurements were done with a 15" diaphragm. Figure 4 represents the K magnitude $(2.2 \mu)$ of NGC 1068 as a function of the position of the center of the galaxy along the diameter of a circular diaphragm of size of $15^{\prime \prime}$, computed by Mr. L. Thompson on the basis of Dr. Neugebauer's results (the K magnitude of NGC 1068 decreased more or less linearly with the logarithm of the diaphragm size from $8.28 \mathrm{mag}$. at $2^{\prime \prime}$ to $7.12 \mathrm{mag}$. at $20^{\prime \prime}$ ). It can be seen from Figure 4 that for any position of the center of NGC 1068 within a 12" circle concentric with the diaphragm, the difference in the $\mathrm{K}$ magnitude is smaller than the total photometric error of measurement of the $\mathrm{K}$ magnitude of a point-like object (star) of com-

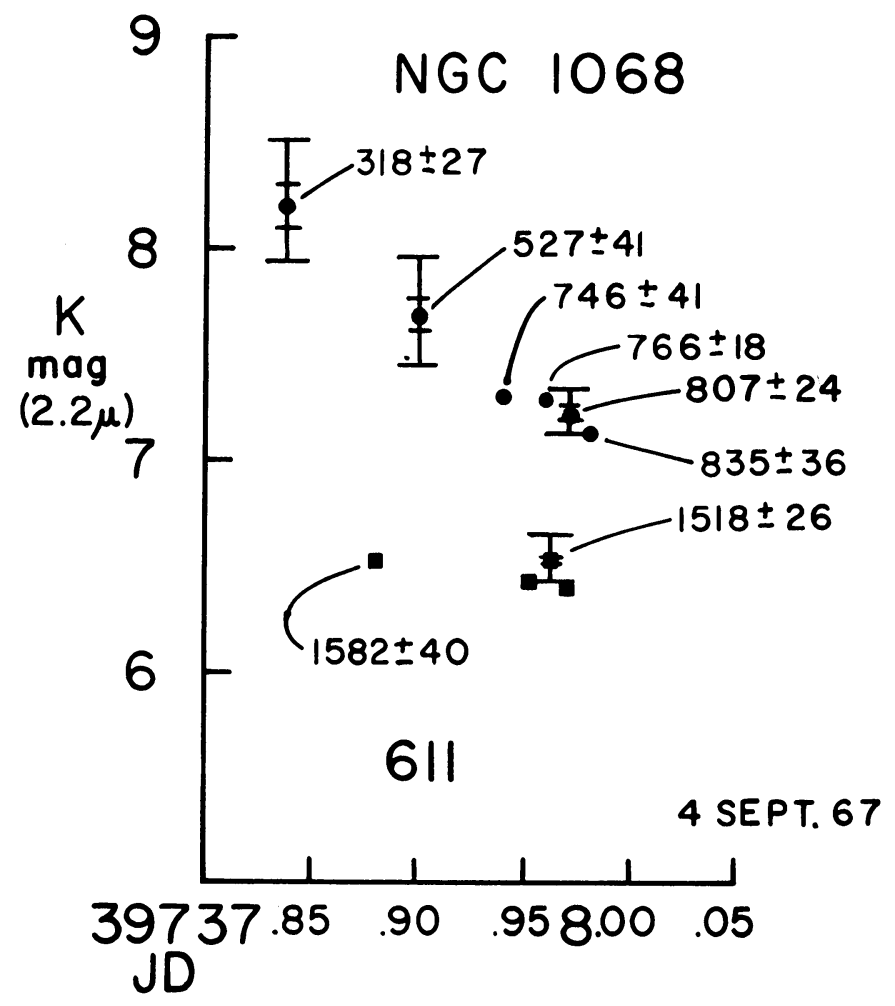

Fig. 5. Observations of NGC 1068 at $2.2 \mu$ on the night of September 4, 1967. The numbers are proportional to average voltages of the photometer signals and their probable errors. The error bars correspond to one (inner bars) and three (outer bars) times the total error of the photometric measurement (in magnitudes).

parable magnitude. Centering errors do not therefore affect significantly the measurements of NGC 1068 and by no means can be responsible for the observed variations of $\mathrm{K}$ magnitudes of this object.

Figure 5, representing an example of observations of NGC 1068 on the night of 
September 4, 1967, confirms the conclusion that the centering errors are not significant. The numbers next to every point in Figure 5 are proportional to average voltages of the photometer signals (each point is an average of 20 differences of readings on 'star' and on 'sky') and their probable errors. The errors are not increasing with decreasing voltage, indicating that the lower average voltages of the upper points are not due to a larger scatter of individual readings (as it would be the case if centering errors were responsible for the lower average), but systematically to lower individual readings. The errors of voltage measurements for NGC 1068 are not larger than those for a point source (comparison star 611, squares in Figure 5). The error bars in Figure 5 represent one (inner bars) and three (outer bars) times the total error of the photometric measurements (in magnitudes, and therefore increasing with increasing magnitude of the object), and include contributions due to extinction and zero point determination.

Note added in proof. In a recent paper Neugebauer et al. (Astrophys. J. Letters 166, L45, 1971) point out that, according to their measurements with diaphragm sizes ranging from $2^{\prime \prime}$ to $20^{\prime \prime}$, the 2.2 micron flux of NGC 1068 can be interpreted as a superposition of a point-like source and a diffuse component. They note that $56 \%$ of the 2.2 micron flux measured by them with a $15^{\prime \prime}$ diaphragm falls outside a 2 " diaphragm and conclude that this is compatible with the variations of flux, observed with a 15" diaphragm, with an amplitude not larger than roughly a factor of two. This limit on amplitude is based on a small number of measurements and its validity rests entirely on the assumption that centering errors at small apertures are negligible. In fact, any errors in centering of such small apertures as $2^{\prime \prime}$ will tend to overestimate the percentage of flux in question and are much more likely to occur with a small diaphragm than with a $15^{\prime \prime}$ diaphragm, even under conditions of good seeing. 